\title{
Preoperative intravenous flurbiprofen reduces postoperative pain and inflammatory cytokines in elderly patients after hip arthroplasty
}

\author{
ZHI-JUN ZHOU*, JIAN TANG* , WEI-HUA LI and WEI-DONG TAO \\ The Second People's Hospital of Nantong, Nantong, Jiangsu 226002, P.R. China
}

Received April 13,2018; Accepted October 11, 2018

DOI: 10.3892/etm.2018.6911

\begin{abstract}
Effect of flurbiprofen on the postoperative cognition and inflammatory cytokines in elderly patients after hip arthroplasty was investigated. Elderly patients undergoing replacement of total hip were randomly divided into three groups of equal size $(n=60)$. A control group (control) received no treatment before or at the end of surgery. The other two groups, PRE and INTRA, received $50 \mathrm{mg}$ flurbiprofen intravenously $15 \mathrm{~min}$ before the surgery and $30 \mathrm{~min}$ before the end of surgery, respectively. The pain score was evaluated by a visual analog scale (VAS) at the following time-points: $24 \mathrm{~h}$ before surgery (T0), and $3 \mathrm{~h}$ (T1), $12 \mathrm{~h}$ (T2) and $24 \mathrm{~h}$ (T3) after surgery. Peripheral venous blood was collected at T0, T1, T2 and T3. Cognitive function was assessed by mini-mental state examination (MMSE) at the time of T0, T1, T2 and T3. The serum concentration of tumor necrosis factor $\alpha$ (TNF- $\alpha$ ), interleukin-1 $\beta$ (IL-1 $\beta$ ), IL-6, and Cox-2 were measured using enzyme linked immunosorbent assay (ELISA). The VAS scores and the release of pro-inflammatory cytokines in the PRE and INTRA groups were less than those in the control group at T1, T2 and T3. The MMSE scores in PRE and INTRA groups were significantly higher than those in the control group at T1, T2 and T3. The PRE group showed a lower VAS score, release of pro-inflammatory cytokines and higher MMSE scores at T1, T2 and T3 than the control and INTRA groups. Intravenous flurbiprofen administration reduces postoperative pain and flammatory response after hip arthroplasty. Moreover, flurbiprofen is more effective when given preoperatively than intraoperatively.
\end{abstract}

Correspondence to: Dr Wei-Hua Li or Dr Wei-Dong Tao, The Second People's Hospital of Nantong, 43 Xinglong Street, Tangzha, Nantong, Jiangsu 226002, P.R. China

E-mail: 1074565829@qq.com

E-mail: weidongtaont@163.com

*Contributed equally

Key words: postoperative cognitive dysfunction, flurbiprofen, preoperative analgesia, inflammatory cytokines

\section{Introduction}

Hip arthroplasty patients suffering from intense pain is thought to prolong mobilization and hospitalization. It would be of value to identify good postoperative analgesia, which may help reduce pain-related multiple problems including anxiety, emergency agitation, and lack of confidence in medical team. Different modes of preoperative, intraoperative and postoperative local analgesic administration have been described in a variety of surgical procedures, such as tangential excision surgery (1), abdominal hysterectomy (2), orthopaedic surgery (3), and thoracotomy (4). However, few studies have described pre- and intraoperative analgesia for hip arthroplasty. Pre-and postoperative administration of valdecoxib reduces the amount of morphine required for postoperative pain relief and provides greater analgesic efficacy compared with morphine alone in orthopedic surgery patients (5). Intraoperative high-volume local infiltration analgesia with ropivacaine $0.2 \%$ provided no additional reduction in acute pain after total hip arthroplasty when combined with oral analgesic regimen consisting of acetaminophen, celecoxib, and gabapentin (6), but more documentation is needed.

Postoperative cognitive decline (POCD) is a common and impactful outcome of surgical procedures in elderly patients (7). It was thought to be associated with cardiac surgery although $40 \%$ of patients were affected after non-cardiac surgical procedures. POCD can last for a long period of time; from a few days to a few years. Patients over 60 reported an incidence of $25.8 \%$ at one week post-surgery and $9.9 \%$ at three weeks post-surgery (8). The pathophysiology and causative mechanisms for POCD are poorly understood. Risk factors include advanced age, preoperative cognitive function, a second operation, length of the operation, respiratory complications and postoperative infection (9). Thus, POCD is an important concern for the patients with surgery.

Inflammatory state characterized by the release of both pro- and anti-inflammatory cytokines is involved in the occurrence of POCD (10). Increased levels of pro-inflammatory cytokines are also associated with a higher incidence of postoperative infections and respiratory failure (11). Considerable evidence suggests a correlation between POCD and factors, such as tumor necrosis factor $\alpha(\mathrm{TNF}-\alpha)$, interleukelin- $1 \beta$ (IL-1ß), and IL-6 $(12,13)$. However, direct evidence showing a 
relationship between POCD and pro-inflammatory cytokines is lamentably absent from the literature.

Nonsteroidal anti-inflammatory drug (NSAID) with high affinity to the site of surgical incision and inflammatory tissues provides postoperative pain relief after different types of surgery (14). The aims of this study were to evaluate the postoperative pain, cognitive function and serum levels of pro-inflammatory cytokines in patients undergoing hip arthroplasty surgery with pre- or intraoperative flurbiprofen.

\section{Patients and methods}

Selection of patients. One hundred and eighty patients undergoing hip arthroplasty surgery from June, 2014 to March, 2016 were involved in this study. The patients were obtained from The Second People's Hospital of Nantong (Nantong, China). The inclusion criteria were: i) American Society of Anesthesiologists (ASA) I-II and ii) age $>70$ years. The exclusion criteria were: i) patients with a history of gastric ulcer and duodenal ulcer, ii) patients with a history of allergic reaction to flurbiprofen, iii) patients with severe hepatic and renal functional disorders, iv) patients with ischemic heart disease, and v) patients with general and local infections.

This randomized and double-blind clinical study was approved by the Ethics Committee of The Second People's Hospital of Nantong, and informed consent was obtained from the patients prior to study enrollment.

Study design. The patients undergoing hip arthroplasty surgery were randomly divided into three groups ( $n=60$ in each group). The control group received no treatment before or at the end of surgery. The PRE group received $50 \mathrm{mg}$ flurbiprofen (Taide Pharmaceutical Co., Beijing, China) intravenously 15 min before surgery. The INTRA group received flurbiprofen $30 \mathrm{~min}$ before the end of surgery.

Anesthesia and analgesia procedures. General anesthesia was induced by intravenous administration of $0.05 \mathrm{mg} / \mathrm{kg}$ midazolam, $0.3 \mu \mathrm{g} / \mathrm{kg}$ sufentanil, $0.3 \mathrm{mg} / \mathrm{kg}$ etomidate, and $0.1 \mathrm{mg} / \mathrm{kg}$ vecuronium bromide. Anesthesia was maintained with continuous infusion of $1-3 \%$ sevoflurane, $0.5-1 \mathrm{mg} / \mathrm{kg} / \mathrm{h}$ propofol, $1-2 \mu \mathrm{g} / \mathrm{kg} / \mathrm{h}$ sufentanil and additional $10 \mu \mathrm{g} / \mathrm{kg}$ atropine and $40 \mu \mathrm{g} / \mathrm{kg}$ neostigmine as required after the surgery. The drug doses were adjusted according to the change of hemodynamics.

Assessment of pain score and cognitive function. A physician who was blinded to the group assignment assessed spontaneous postsurgical pain intensity at rest using a $10-\mathrm{cm}$ visual analog scale (VAS), anchored at 'no pain' and 'worst pain I can imagine', at $24 \mathrm{~h}$ before surgery (T0), and $3 \mathrm{~h}$ (T1), $12 \mathrm{~h}$ (T2) and $24 \mathrm{~h}$ (T3) after surgery. Another physician who was blinded to the group assignment assessed cognitive function at T0, T1, T2 and T3 using mini-mental state examination (MMSE). A score of 24 out of a possible 30 was considered the cut-off score for POCD (15).

Determination of the levels of systemic pro-inflammatory cytokines. Blood samples were collected at the time of T0, $\mathrm{T} 1, \mathrm{~T} 2$ and T3 to measure serum TNF- $\alpha$, IL-1 $\beta$, IL-6, and
Table I. General data of the three groups (mean \pm standard deviation).

\begin{tabular}{lccc}
\hline Items & $\begin{array}{c}\text { Control } \\
(\mathrm{n}=60)\end{array}$ & $\begin{array}{c}\text { PRE } \\
(\mathrm{n}=60)\end{array}$ & $\begin{array}{c}\text { INTRA } \\
(\mathrm{n}=60)\end{array}$ \\
\hline Sex (F/M) & $28 / 32$ & $22 / 38$ & $24 / 36$ \\
Age (years) & $75.2 \pm 5.3$ & $78.3 \pm 6.1$ & $76.1 \pm 5.9$ \\
Weight (kg) & $59.6 \pm 8.7$ & $61.5 \pm 5.4$ & $62.8 \pm 7.6$ \\
Height (cm) & $164.3 \pm 7.5$ & $162.8 \pm 5.8$ & $161.9 \pm 6.4$ \\
Operation time (min) & $118.3 \pm 20.5$ & $123.7 \pm 22.9$ & $112.1 \pm 24.7$ \\
\hline
\end{tabular}

Table II. VAS score among the three groups (mean \pm standard deviation).

\begin{tabular}{llll}
\hline Items & $\begin{array}{c}\text { Control } \\
(\mathrm{n}=60)\end{array}$ & $\begin{array}{c}\text { PRE } \\
(\mathrm{n}=60)\end{array}$ & $\begin{array}{c}\text { INTRA } \\
(\mathrm{n}=60)\end{array}$ \\
\hline T0 & $1.0 \pm 0.5$ & $1.3 \pm 0.2$ & $1.5 \pm 0.3$ \\
T1 & $5.9 \pm 0.8$ & $3.2 \pm 0.4^{\mathrm{b}, \mathrm{c}}$ & $4.3 \pm 0.5^{\mathrm{a}}$ \\
$\mathrm{T} 2$ & $4.8 \pm 0.5$ & $2.7 \pm 0.4^{\mathrm{b}, \mathrm{c}}$ & $3.5 \pm 0.2^{\mathrm{a}}$ \\
T3 & $3.5 \pm 0.5$ & $1.8 \pm 0.3^{\mathrm{b}, \mathrm{c}}$ & $2.5 \pm 0.3^{\mathrm{a}}$ \\
\hline
\end{tabular}

${ }^{\mathrm{a}} \mathrm{P}<0.05,{ }^{\mathrm{b}} \mathrm{P}<0.01$ vs. control group; ${ }^{\mathrm{c}} \mathrm{P}<0.05$ vs. INTRA group. VAS, visual analog scale.

cyclooxygenase-2 (Cox-2) levels. Peripheral venous blood samples were collected and then centrifuged at $1,000 \mathrm{x} \mathrm{g}$ at $4^{\circ} \mathrm{C}$ for $10 \mathrm{~min}$, and serum samples were obtained. Serum pro-inflammatory cytokines TNF- $\alpha$, IL-1 $\beta$, IL- 6 , and Cox-2 levels were measured, using the commercially available human ELISA kit (Jiancheng Technology Co., Ltd., Nanjing, China) as described. All steps involved were according to the manufacturers' protocol.

Statistical analysis. Parametric results were expressed as mean \pm standard deviation for each group. Statistical analysis was performed with SPSS software, version 17.0 (SPSS, Inc., Chicago, IL, USA). Student's t-test and one-way ANOVA followed by Turkey's post hoc test were used for the assessment among the groups. $\mathrm{P}<0.05$ was considered to indicate a statistically significant difference.

\section{Results}

Patient characteristics. In total, 180 patients were involved in the present study. As shown in Table I, there were no significant differences in the three groups in terms of sex, age, weight, height, and operation time.

VAS scores. VAS data are presented in Table II. The results showed there were no significant differences among the control, PRE and INTRA groups at $24 \mathrm{~h}$ before surgery (T0). VAS scores in groups PRE and INTRA were significantly decreased compared to that in control group at 3 (T1), 12 (T2) and $24 \mathrm{~h}$ (T3) after surgery, and the VAS score in the PRE 
A

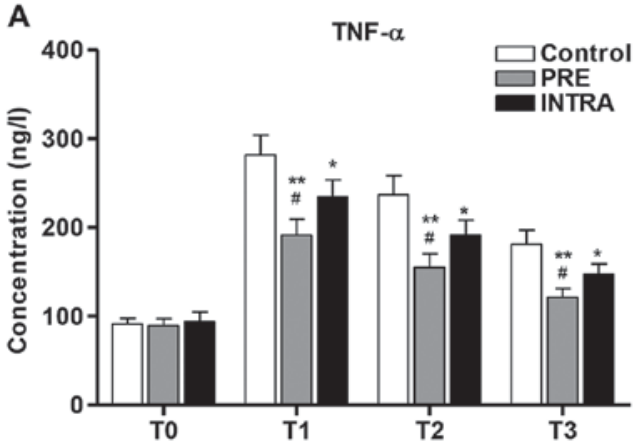

C

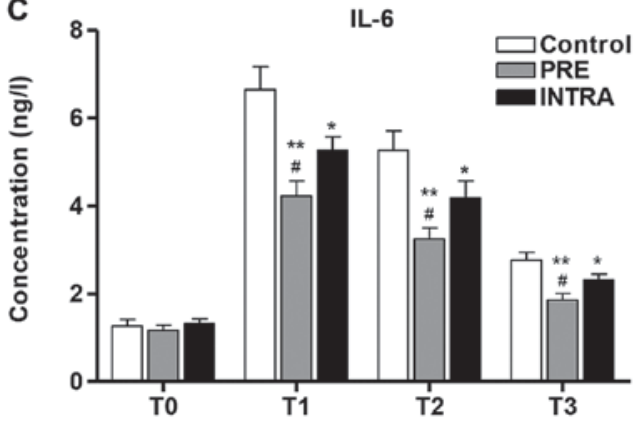

B

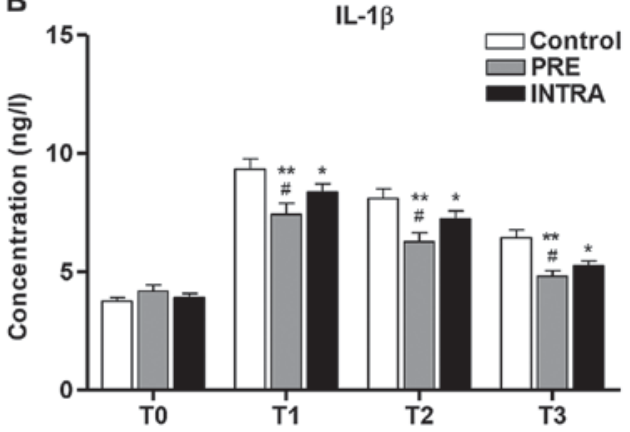

D

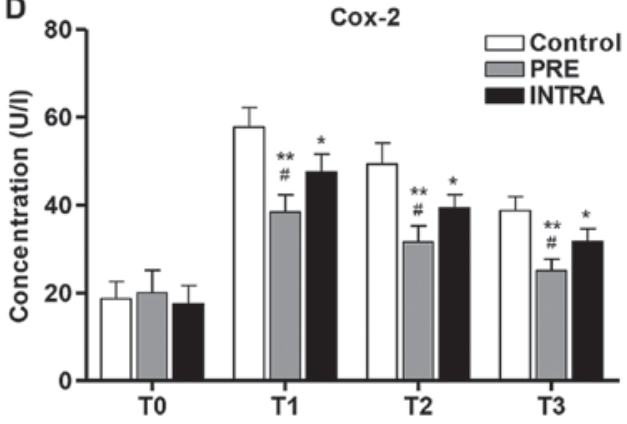

Figure 1. Serum levels of pro-inflammatory cytokines among the three groups (mean \pm standard deviation). Serum levels of pro-inflammatory cytokines including TNF- $\alpha$ (A), IL-1 $\beta$ (B), IL-6 (C) and Cox-2 (D) were measured by ELISA. ${ }^{*} \mathrm{P}<0.05,{ }^{* * *} \mathrm{P}<0.01$ vs. control group; ${ }^{*} \mathrm{P}<0.05$ vs. INTRA group. TNF- $\alpha$, tumor necrosis factor $\alpha$; IL-1 $\beta$, interleukin-1 $\beta$; Cox-2, cyclooxygenase-2.

Table III. MMSE score in the three groups (mean \pm standard deviation).

\begin{tabular}{lccc}
\hline Items & $\begin{array}{c}\text { Control } \\
(\mathrm{n}=60)\end{array}$ & $\begin{array}{c}\text { PRE } \\
(\mathrm{n}=60)\end{array}$ & $\begin{array}{c}\text { INTRA } \\
(\mathrm{n}=60)\end{array}$ \\
\hline T0 & $28.9 \pm 1.2$ & $29.5 \pm 0.8$ & $28.1 \pm 1.4$ \\
T1 & $20.4 \pm 0.8$ & $24.8 \pm 0.9^{\mathrm{b}, \mathrm{c}}$ & $23.1 \pm 0.5^{\mathrm{a}}$ \\
T2 & $21.1 \pm 0.9$ & $26.8 \pm 1.3^{\mathrm{b}, \mathrm{c}}$ & $24.1 \pm 0.8^{\mathrm{a}}$ \\
T3 & $22.8 \pm 1.3$ & $28.8 \pm 1.4^{\mathrm{b}, \mathrm{c}}$ & $25.7 \pm 1.2^{\mathrm{a}}$ \\
\hline
\end{tabular}

${ }^{a} \mathrm{P}<0.05,{ }^{b} \mathrm{P}<0.01$ vs. control group; ${ }^{\mathrm{c}} \mathrm{P}<0.05$ vs. INTRA group. MMSE, mini-mental state examination.

group was significantly lower than that in the INTRA group at the time of $\mathrm{T} 1, \mathrm{~T} 2$ and $\mathrm{T} 3$.

MMSE scores. To further investigate the efficacy of flurbiprofen, we assessed the cognitive function by MMSE. As shown in Table III, there was no significant difference among the three groups at T0. The MMSE scores at T1, T2 and T3 in PRE and INTRA group were significantly higher than that in the control group, and the MMSE score in the PRE group was significantly higher than that in the INTRA group at the time of $\mathrm{T} 1, \mathrm{~T} 2$ and $\mathrm{T} 3$.

Serum levels of pro-inflammatory cytokines. We investigated the anti-inflammatory effects of flurbiprofen in patients undergoing hip arthroplasty surgery. As shown in Fig. 1A-D, postoperative increases in serum TNF- $\alpha$, IL-1 $\beta$, IL- 6 and
Cox-2 concentrations were observed among the three groups. Serum TNF- $\alpha$, IL-1 $\beta$, IL- 6 and Cox-2 levels did not differ significantly in T0 among the three groups. However, TNF- $\alpha$, IL-1 $\beta$, IL- 6 and Cox-2 levels were significantly decreased in the PRE and INTRA groups compared to that in the control group, and the pro-inflammatory cytokines were significantly lower in the PRE group than that in the INTRA group at the time of $\mathrm{T} 1, \mathrm{~T} 2$ and $\mathrm{T} 3$.

\section{Discussion}

Previous studies reported attentional dysfunction as sensitive and prominent characteristics of POCD (16). The clinical significance of POCD is evidenced by the impact that daily activities confers particularly on the elderly patients (17). The cohort study confirms that there is a significantly increased incident of cognitive dysfunction in patients over 70 undergoing non-cardiac surgery, and emphasized by the evidence that preoperative or intraoperative intravenous administration of flurbiprofen reduces the level of cognitive dysfunction at 3,12 and $24 \mathrm{~h}$ after hip arthroplasty surgery. In line with our findings that a greater level of cognitive dysfunction is still observed after 52 weeks in people over 60 undergoing abdominal or orthopaedic surgery in comparison to agematched controls (3). Despite the persistent impact of POCD on mortality and welfare of patients was not included in the present study the cognitive impairment was indeed observed during the period of $24 \mathrm{~h}$ after surgery, highlighting the clinical importance of POCD and its long time impact on cognition in the patients with non-cardiac surgery.

VAS scoring is a simple and commonly used method for evaluating variations in pain intensity in the early postoperative 
period. VAS scoring was used for evaluating postoperative pain in different studies, including total hip arthroplasty (14), spinal fusion (18), abdominal (19), and minor gynecological surgery (20). In the present study, postoperative VAS scores were significantly increased compared with preoperative controls, while preoperative or intraoperative flurbiprofen administration reduced postoperative pain induced by surgery after 3,12 and $24 \mathrm{~h}$, which is in line with a previous study (21). MMSE is a brief test of cognitive impairment used widely to screen for dementia. The original test, developed by Folstein et al, includes questions about orientation, attention, recall, and language (22). In the present study, the MMSE measure was used to analyze the level of cognitive decline and similar to the previous study that postoperative MMSE scores were significantly decreased compared with preoperative controls, while preoperative or intraoperative flurbiprofen administration improved POCD evidenced by increased MMSE scores induced by surgery after 3, 12 and $24 \mathrm{~h}$ (23).

Pro-inflammatory cytokine response induced by surgical damage has been reported and is thought to provide a source of postoperative sensory signals and induce central sensitization (24). Moreover, a meta-analysis strengthens the clinical evidence that POCD is accompanied by a peripheral inflammatory reaction (13). IL-6 and TNF- $\alpha$ are major mediators of the acute-phase protein response to tissue damage caused by surgical intervention. Furthermore, it has been reported that postoperative IL-6 levels correlate with postoperative complications and mortality, and might have contribution to the occurrence of POCD (13). IL-1 $\beta$ is a pro-inflammatory cytokine that contributes to neuro-inflammation in many central nervous system (CNS) disorders (25). Studies have shown that Cox-2 inhibitor impeded neuro-inflammation and induced amelioration of cognitive function in patients with Alzheimer disease, suggesting a critical role of Cox-2 in driving the pathogenesis of cognitive decline after surgery (26). In the present study, the concentrations of TNF- $\alpha$, IL-1 $\beta$, IL- 6 and Cox- 2 increased significantly in the patients after hip arthroplasty surgery compared to before. There was a significant increase in TNF- $\alpha$, IL-1 $\beta$, IL-6 and Cox- 2 concentration at $3 \mathrm{~h}$ after surgery, although IL-1 $\beta$ protein was significantly increased at $24 \mathrm{~h}$ after surgery and no increase in protein expression of TNF- $\alpha$ (27). Importantly, preoperative or intraoperative flurbiprofen administration reduced the increase in the concentrations of TNF- $\alpha$, IL-1 $\beta$, IL- 6 and Cox-2 induced by surgery after 3, 12 and $24 \mathrm{~h}$.

Although some clinical studies have conflicting results regarding the efficacy of preemptive analgesia, it is to give a first dose of analgesics before pain stimulation and has been regarded as a new concept to enhance the postoperative analgesia. Flurbiprofen, an injectable NSAID, is an effective and safe analgesic providing postoperative pain relief after different surgery. However, the efficacy of pre-emptive analgesia with NSAID remains disputed. Preoperative administration of piroxicam sublingually would reduce postoperative pain and opioid requirements more than would postoperative piroxicam (28). Nimesulide is a well-tolerated oral anti-inflammatory drug with a superior analgesic activity compared with naproxen and placebo after out-patient orthopedic surgery (29). Moreover, a combination of paracetamol and an NSAID may offer superior analgesia compared with either drug alone (30). Intravenous flurbiprofen reduces postoperative rescue analgesic requirement after abdominal hysterectomy, and is more effective when given before than after surgery (2). In the present study, we consider that flurbiprofen reduced the postoperative pain and the occurrence of POCD evidenced by increased MMSE scores after surgery through preoperative or intraoperative administration. Importantly flurbiprofen is more effective when given preoperatively than intraoperatively. This is the first report that intraoperatively administrated flurbiprofen confers a postoperative pain relief for hip arthroplasty surgery, although the efficacy is less than preoperatively.

Other anti-postoperative pain effect of flurbiprofen, antiinflammatory response was also found in variety of studies and correlated with the occurrence of POCD. Zhang et al found that preoperative flurbiprofen can effectively inhibit pro-inflammatory cytokines, maintain the balance of cytokines and reduce myocardiac injury, and be beneficial to patients with early rehabilitation after operation (31). Thus, we speculated that flurbiprofen is a suitable NSAID to induce a preemptive effect. Geng et al found that preoperative administration of flurbiprofen reduced the plasma levels of TNF- $\alpha$ and IL-6, and was further reduced by postoperative administration of flurbiprofen at 24 and $48 \mathrm{~h}$ after the operation (1). Consistent with the above findings, we found that preoperative or intraoperative administration of flurbiprofen, especially intraoperative flurbiprofen significantly decreased the concentrations of TNF- $\alpha$, IL-1 $\beta$, IL- 6 and Cox- 2 at 3, 12, and $24 \mathrm{~h}$ after surgery, indicating that analgesia with anti-inflammatory drug may contribute to the attenuation of the postoperative inflammatory response and prevent postoperative pain in patients undergoing hip arthroplasty.

We conclude that the preoperative or intraoperative intravenous administration of flurbiprofen is effective in reducing postoperative pain and the occurrence of POCD, as well as the concentrations of pro-inflammatory cytokines. Moreover, flurbiprofen is more effective when given preoperatively than intraoperatively.

\section{Acknowledgements}

Not applicable.

\section{Funding}

No funding was received.

\section{Availability of data and materials}

The datasets used and/or analyzed during the present study are available from the corresponding author on reasonable request.

\section{Authors' contributions}

JT and WHL performed experiments and data analysis; and ZJZ and WDT designed the project and wrote the study. All authors read and approved the study.

\section{Ethics approval and consent to participate}

The study was approved by the Ethics Committee of the Second People's Hospital of Nantong (Nantong, China). 
Signed informed consents were obtained from the patients or the guardians.

\section{Patient consent for publication}

Not applicable.

\section{Competing interests}

The authors declare that they have no competing interests.

\section{References}

1. Geng W, Hong W, Wang J, Dai Q, Mo Y, Shi K, Sun J, Qin J, Li M and Tang H: Flurbiprofen axetil enhances analgesic effects of sufentanil and attenuates postoperative emergence agitation and systemic proinflammation in patients undergoing tangential excision surgery. Mediators Inflamm 2015: 601083, 2015.

2. Nakayama M, Ichinose $H$, Yamamoto $S$, Nakabayashi $K$, Satoh O and Namiki A: Perioperative intravenous flurbiprofen reduces postoperative pain after abdominal hysterectomy. Can J Anaesth 48: 234-237, 2001

3. Ballard C, Jones E, Gauge N, Aarsland D, Nilsen OB, Saxby BK, Lowery D, Corbett A, Wesnes K, Katsaiti E, et al: Optimised anaesthesia to reduce post operative cognitive decline (POCD) in older patients undergoing elective surgery, a randomised controlled trial. PLoS One 7: e37410, 2012.

4. Esme H, Kesli R, Apiliogullari B, Duran FM and Yoldas B: Effects of flurbiprofen on CRP, TNF- $\alpha$, IL-6, and postoperative pain of thoracotomy. Int J Med Sci 8: 216-221, 2011.

5. Camu F, Beecher T, Recker DP and Verburg KM: Valdecoxib, a COX-2-specific inhibitor, is an efficacious, opioid-sparing analgesic in patients undergoing hip arthroplasty. Am J Ther 9: 43-51, 2002.

6. Lunn TH, Husted H, Solgaard S, Kristensen BB, Otte KS, Kjersgaard AG, Gaarn-Larsen L and Kehlet H: Intraoperative local infiltration analgesia for early analgesia after total hip arthroplasty: A randomized, double-blind, placebo-controlled trial. Reg Anesth Pain Med 36: 424-429, 2011.

7. Silbert B,Evered L and Scott DA: Cognitive decline in the elderly: Is anaesthesia implicated? Best Pract Res Clin Anaesthesiol 25: 379-393, 2011

8. Moller JT, Cluitmans P, Rasmussen LS, Houx P, Rasmussen H, Canet J, Rabbitt P, Jolles J, Larsen K, Hanning CD, et al; International Study of Post-Operative Cognitive Dysfunction: Long-term postoperative cognitive dysfunction in the elderly ISPOCD1 study. ISPOCD investigators. Lancet 351: 857-861, 1998.

9. Newman S, Stygall J, Hirani S, Shaefi S and Maze M: Postoperative cognitive dysfunction after noncardiac surgery: A systematic review. Anesthesiology 106: 572-590, 2007.

10. Fidalgo AR, Cibelli M, White JP, Nagy I, Maze M and Ma D: Systemic inflammation enhances surgery-induced cognitive dysfunction in mice. Neurosci Lett 498: 63-66, 2011.

11. Takeda S, Takeda S, Kim C, Ikezaki H, Nakanishi K, Sakamoto A, Okawa K, Miyashita M, Sasajima K, Tajiri T, et al: Preoperative administration of methylprednisolone attenuates cytokine-induced respiratory failure after esophageal resection. J Nippon Med Sch 70: 16-20, 2003.

12. Cibelli M,Fidalgo AR, Terrando N,MaD,Monaco C,Feldmann M, Takata M, Lever IJ, Nanchahal J, Fanselow MS, et al: Role of interleukin-1 $\beta$ in postoperative cognitive dysfunction. Ann Neurol 68: 360-368, 2010.

13. Peng L, Xu L and Ouyang W: Role of peripheral inflammatory markers in postoperative cognitive dysfunction (POCD): A meta-analysis. PLoS One 8: e79624, 2013.

14. Andersen LJ, Poulsen T, Krogh B and Nielsen T: Postoperative analgesia in total hip arthroplasty: A randomized double-blinded, placebo-controlled study on peroperative and postoperative ropivacaine, ketorolac, and adrenaline wound infiltration. Acta Orthop 78: 187-192, 2007.
15. Galea M and Woodward M: Mini-mental state examination (MMSE). Aust J Physiother 51: 198, 2005.

16. van Dijk D, Keizer AM, Diephuis JC, Durand C, Vos LJ and Hijman R: Neurocognitive dysfunction after coronary artery bypass surgery: A systematic review. J Thorac Cardiovasc Surg 120: 632-639, 2000.

17. Kojima Y and Narita M: Postoperative outcome among elderly patients after general anesthesia. Acta Anaesthesiol Scand 50: 19-25, 2006.

18. Yamashita K, Fukusaki M, Ando Y, Fujinaga A, Tanabe T, Terao Y and Sumikawa K: Preoperative administration of intravenous flurbiprofen axetil reduces postoperative pain for spinal fusion surgery. J Anesth 20: 92-95, 2006.

19. McDonnell JG, O'Donnell B, Curley G, Heffernan A, Power C and Laffey JG: The analgesic efficacy of transversus abdominis plane block after abdominal surgery: A prospective randomized controlled trial. Anesth Analg 104: 193-197, 2007.

20. Paech MJ, Goy R, Chua S, Scott K, Christmas T and Doherty DA: A randomized, placebo-controlled trial of preoperative oral pregabalin for postoperative pain relief after minor gynecological surgery. Anesth Analg 105: 1449-1453, 2007.

21. Takada M, Fukusaki M, Terao Y, Yamashita K, Inadomi C, Takada $\mathrm{M}$ and Sumikawa K: Preadministration of flurbiprofen suppresses prostaglandin production and postoperative pain in orthopedic patients undergoing tourniquet inflation. J Clin Anesth 19: 97-100, 2007.

22. Folstein MF, Folstein SE and McHugh PR: 'Mini-mental state'. A practical method for grading the cognitive state of patients for the clinician. J Psychiatr Res 12: 189-198, 1975.

23. Kan ZY, Hu J and Wang F: Effect of Flurbiprofen on postoperative cognition and inflammatory cytokines in elderly male patients. Practical J Clin Med 2: 81-83, 2012 (In Chinese).

24. Rosczyk HA, Sparkman NL and Johnson RW: Neuroinflammation and cognitive function in aged mice following minor surgery. Exp Gerontol 43: 840-846, 2008.

25. Wu MD, Montgomery SL, Rivera-Escalera F, Olschowka JA and O'Banion MK: Sustained IL-1 $\beta$ expression impairs adult hippocampal neurogenesis independent of IL-1 signaling in nestin ${ }^{+}$ neural precursor cells. Brain Behav Immun 32: 9-18, 2013.

26. Peng M, Wang YL, Wang FF, Chen $C$ and Wang CY: The cyclooxygenase-2 inhibitor parecoxib inhibits surgery-induced proinflammatory cytokine expression in the hippocampus in aged rats. J Surg Res 178: e1-e8, 2012.

27. Wan Y, Xu J, Ma D, Zeng Y, Cibelli M and Maze M: Postoperative impairment of cognitive function in rats: A possible role for cytokine-mediated inflammation in the hippocampus. Anesthesiology 106: 436-443, 2007.

28. Gramke HF, Petry JJ, Durieux ME, Mustaki JP, Vercauteren M, Verheecke G and Marcus MA: Sublingual piroxicam for postoperative analgesia: preoperative versus postoperative administration: a randomized, double-blind study. Anesth Analg 102: 755-758, 2006.

29. Binning A: Nimesulide in the treatment of postoperative pain: A double-blind, comparative study in patients undergoing arthroscopic knee surgery. Clin J Pain 23: 565-570, 2007.

30. Ong CK, Seymour RA, Lirk P and Merry AF: Combining paracetamol (acetaminophen) with nonsteroidal antiinflammatory drugs: A qualitative systematic review of analgesic efficacy for acute postoperative pain. Anesth Analg 110: 1170-1179, 2010.

31. Zhang Y, Gu E and Liu X: Effects of flurbiprofen axetil on inflammatory cytokines and myocardiac injury in patients undergoing cardiac valve replacement surgery with cardiopulmonary bypass. Acta Univ Med Anhui 1: 92-95, 2009 (In Chinese).

This work is licensed under a Creative Commons Attribution-NonCommercial-NoDerivatives 4.0 International (CC BY-NC-ND 4.0) License. 